\title{
La chair ouverte à la portance de l'être
}

\section{Emmanuel de Saint Aubert}

\section{OpenEdition}

\section{Journals}

Édition électronique

URL : http://journals.openedition.org/alter/384

DOI : 10.4000/alter.384

ISSN : 2558-7927

\section{Éditeur :}

Association ALTER, Archives Husserl (CNRS-UMR 8547)

\section{Édition imprimée}

Date de publication : 1 novembre 2015

Pagination : 168-185

ISBN : 978-2-9550449-1-9

ISSN : 1249-8947

Référence électronique

Emmanuel de Saint Aubert, «La chair ouverte à la portance de l'être », Alter [En ligne], 23 | 2015, mis en ligne le 01 décembre 2017, consulté le 01 mai 2019. URL : http://journals.openedition.org/alter/384 ; DOI : 10.4000/alter.384 


\section{LA CHAIR OUVERTE À LA PORTANCE DE L'ÊTRE}

Emmanuel de Saint Aubert

L'être humain est une manière singulière d'être corps, en rapport au monde, en relation avec autrui. Ce style qui constitue sa chair s'exprime dans certaines attitudes typiques marquées par une conjonction, parfois extrême, de passivité et d'activité - dès l'amplitude comme la profondeur de sa perception du monde, dans l'accueil et l'écoute qu'il peut accorder à autrui, dans une posture foncièrement interrogative intriquée avec des dimensions de consentement et de foi. Ces attitudes montrent en lui une étonnante capacité à s'ouvrir, à éprouver et mettre à l'épreuve, recevoir et se donner dans un même geste ; à se laisser traverser et configurer par les structures de l'autre, tout en lui prêtant ou lui imposant ses propres structures. Sa chair porte aux tréfonds de sa contexture la marque d'incessantes incorporations, tapissée qu'elle est de ce qu'elle perçoit, connaît et désire, tout en habillant perpétuellement d'elle-même ce avec quoi et avec qui elle entre en relation.

Dans cette manière d'être corps ouvert, chair étoilée en compassion avec ce qu'elle perçoit et désire, l'être humain n'est pas qu'en rapport avec le monde et avec autrui. Au cœur même de ces relations, il s'ouvre aussi à une indétermination aux visages multiples. Et son endurance de l'indétermination, qui travaille notamment sa foi, son espérance et son désir, est une condition de possibilité de son rapport au monde et à autrui - sans elle, ces relations sont vouées à l'échec. Notre rapport à cette indétermination polymorphe - de l'abîme à la profondeur, du vide au débordement, de l'inaccessible à l'inépuisable, du non-sens au mystère - est lui-même polymorphe - de la chute au relèvement, du vertige à l'envol, de l'angoisse au désir. Il est au cœur de la condition humaine, de ce avec quoi et parfois contre quoi nous nous battons, dans les nœuds clandestins du tragique et de 
l'espoir, au risque du plus misérable et du plus grand de ce que nous pouvons devenir.

Les visages si divers, presque contradictoires, que peut prendre l'indétermination, offrent une esquisse privilégiée de ce que nous nommerons l'être, dans une approche négative qui distingue ce dernier de l'être en tant qu'étant ceci ou cela, revêtu de tel ou tel attribut, de telle ou telle détermination. Et notre ouverture à l'indétermination constitue ce que nous nommerons la dimension existentielle de certaines attitudes humaines. L'existentialisme est un humanisme, à ceci près que la question de l'homme y connaît une essentielle décentration, en étant axée, à travers et au-delà de son rapport au monde et à autrui, sur son ouverture à l'être. D'où un lien inextricable entre anthropologie et ontologie, celle-ci marquant le nécessaire accomplissement de celle-là et non pas un dépassement qui la nierait ou la quitterait pour d'autres contrées. Nous en situons le croisement essentiel dans une phénoménologie de notre ouverture complexe à l'indétermination, qui sache être attentive aux modalités d'animation et d'expression les plus élémentaires de notre institution corporelle. Des modalités toujours travaillées, dans les fondements mêmes de notre être-au-monde, par les attentes et enjeux du rapport à autrui, par une épreuve de la portance des êtres et de la portance de l'être ${ }^{1}$.

\section{L'indétermination de l'être}

Nous venons d'introduire une première formulation de notre rapport à l'être comme rapport à l'indétermination. Il faut aussitôt étoffer et différencier cette notion, qui renvoie à une absence relative ou totale de déterminations (l'indéterminé), à laquelle peut être associée une impossibilité à déterminer (l'indéterminable); impossibilité à déterminer tant ce qui échappe radicalement à nos capacités de détermination (l'inaccessible) que ce que nous n'aurons jamais fini de déterminer, notamment en raison d'une prodigalité débordante de déterminations (l'inépuisable) - cette dernière direction de sens venant en partie renverser la première, puisqu'ici l'absence ou le vide (de déterminations) cède la place à la surabondance voire à l'infini.

\footnotetext{
${ }^{1}$ Le texte ici proposé est le reflet d'une réflexion en cours sur la notion de "portance ", travaillée dans divers champs cliniques, notamment avec des psychologues cliniciens et des enseignants spécialisés. Cet article se concentre sur certains aspects théoriques de cette notion ; pour quelques aperçus sur ses sources et enjeux cliniques, nous nous permettons de renvoyer à un texte à paraître dans les Archives de philosophie.
} 
Aux prises avec cette indétermination polymorphe, l'être humain connait autant de vertiges correspondants, qui peuvent sembler contraires : entre le vide du gouffre et l'inépuisable richesse de la profondeur, entre le mur impénétrable du pur non-sens et l'ouverture d'un mystère que l'on n'aura jamais fini de percevoir, percer et comprendre. Pourtant la frontière entre ces contraires est parfois ténue, et les vertiges de l'indétermination ne sont pas toujours incompatibles. Tel le parachutiste qui expérimente la chute dans l'abîme et la portance de l'air, tout être humain est amené à vivre les passages ambigus du vertige au soutien, de ce qui écrase et déprime l'action à ce qui l'encourage et la remet en tension vers son but. Passages ambigus entre deux manières d'être au monde apparemment contraires, mais aussi mélanges détonnants: celui qui saute dans le vide apparent pour en retrouver et éprouver l'élément porteur, connaît parfois une conjonction extrême d'angoisse et de désir. Nous avons tous l'occasion de vivre cette même conjonction, dans notre rapport à l'invisible et l'imprévisible, à l'inconnu, à autrui.

L'être humain est un funambule de l'absence et de la présence, de l'angoisse et du désir, du non-sens et du mystère. Il passe son existence à tenter de passer d'un bord à l'autre des virtualités contraires de l'indétermination, n'y parvient pas toujours, il s'en faut, et n'y parvient jamais seul. Mais quoi qu'il arrive, les deux bords sont secrètement liés. À vouloir à tout prix supprimer l'absence, l'angoisse et le non-sens, du moins en soustraire l'être humain, l'en protéger par telle ou telle muraille idéologique ou psychologique, technologique ou chimique - nous sommes aujourd'hui parvenus à une remarquable combinaison des quatre -, on le prive étrangement de quelques ressorts essentiels d'une véritable ouverture à la présence, de la croissance d'un désir libre, et de l'endurance du mystère.

Ces directions de sens de l'indétermination et de notre rapport avec elle ont été en partie frayées par Merleau-Ponty, à partir d'une phénoménologie de la vie perceptive, au fil de l'élaboration de son anthropologie, et plus encore dans l'accomplissement ontologique de celle-ci. En partie seulement, donc de manière lacunaire, mais suffisante pour y trouver appui dans la poursuite d'un approfondissement du sens et des sens de l'être. À n'en pas douter, Merleau-Ponty est spontanément plus attiré par la profondeur que par le vertige, par l'inépuisable que par l'inaccessible, et s'il parle du non-sens, il semble toujours le tirer du côté du mystère. Les figures merleau-pontiennes de l'être nous permettent ainsi de mieux comprendre les modalités fécondes de notre ouverture à une indétermination " positive ». Elles donnent à l'être divers visages plus ou moins sensibles, qui ne caté- 
gorisent pas cette notion si délicate mais la colorent d'autant de dimensions transversales, sinon analogiques. Nous privilégierons ces métaphores, qui permettent d'entrevoir que notre être-au-monde, dès la perception, ne s'arrête pas au monde mais s'ouvre à l'être, cet être à l'être se jouant à même notre être-au-monde.

L'ontologie de Merleau-Ponty cheminait notamment dans un grand œuvre resté inachevé dont l'intitulé était tour à tour Être et Monde et Le visible et l'invisible, associant d'un côté le visible et le monde et, de l'autre, l'invisible et l'être. Sa phénoménologie le conduit à discerner que notre être-au-monde, en accédant au monde et à autrui - et pour pouvoir y accéder -, va à travers eux au-delà d'eux, s'ouvre à un inaccessible sans pour autant le posséder, s'ouvre à un inépuisable sans pour autant l'épuiser. Se jouent ici à la fois la transcendance de notre chair et une condition de possibilité de son accès aux choses mêmes, son ouverture à l'horizon inaccessible et son accès à une profondeur sans laquelle elle ne saurait percevoir, selon une dialectique subtile de l'accessible et de l'inaccessible que Merleau-Ponty fraye dans son traitement original de la notion $\mathrm{d}^{\prime}$ « ultra-chose $»^{2}$.

L'être s'annonce ainsi sous les diverses inscriptions de l'invisible merleau-pontien. Il est fond, le fond de (la figure), ce sur fond de quoi chaque chose (à commencer par autrui) se distingue, et émerge ainsi à son identité. Profondeur commune des êtres, il est ce qui est entre (les choses), les écarte et les relie à la fois. Il est le silence du monde, non pas d'abord comme privation de bruit ou de parole, mais là aussi au sens de ce qui est entre (les paroles), et qui fait fond (de la parole et de la relation). Il est l'ombre des choses, caché et figuratif, latence qui porte le visible et le rend visible. Il est envers de la corporéité et de l'intercorporéité, envers des êtres et de leurs relations, incorporel qui habite le désir de la chair jusqu'au corps glorieux de la parole ${ }^{3}$. L'être est ainsi fort d'une double virtualité aux enjeux anthropologiques majeurs : il permet aux êtres de trouver leur identité, il soutient leur relation. Et l'un par l'autre. Car si l'être est tiers, il n'est pas seulement, ni même d'abord, tiers séparé et séparateur, mais tiers inclus, enveloppant-enveloppé et engendrant-engendré de nos liens. Identité et relation sont typiquement indissociables dans l'ontologie comme dans l'anthropologie de Merleau-Ponty. Avec une conséquence impli-

\footnotetext{
${ }^{2}$ À ce sujet, voir notre contribution «Au croisement du réel et de l'imaginaire : les "ultrachoses" chez Merleau-Ponty", in Is this real ? Phenomenologies of the imaginary, A. Dufourcq et K. Novotný (éd.), Brill, à paraître en 2016.

${ }^{3}$ Sur ces différentes esquisses de l'être, voir notre ouvrage Être et chair I, Paris, Vrin, 2013, chapitre IX.
} 
cite : l'éthique est greffée sur l'ontologie, tout en s'ouvrant d'emblée avec elle.

Abordé dans la cohérence de cet univers métaphorique, l'être n'apparaît pas uniquement, ni même d'abord, comme ce qui se tient mais ce qui nous tient. Il n'est pas d'abord ce qui se maintient, dans l'intégrité de soi, en deçà des accidents, malgré l'adversité, les pressions et le changement - la substance. Mais ce qui tient ensemble les choses et ce qui nous tient ensemble - la portance de notre coexistence ${ }^{4}$. L'être s'annonce moins comme ce qui résiste (en soi) que comme ce qui nous résiste - telle la Nature, dans la façon dont Merleau-Ponty introduisait cette notion contre la liberté absolue sartrienne ${ }^{5}$. Il $n^{\prime}$ est pas un arrière-monde, mais la profondeur de ce monde, infrastructure et fond, qui ne se tient pas dans un ailleurs, mais entre les êtres et au cœur même de leurs relations. Il n'est pas séparé, par une différence irréductible qu'il s'agirait de transgresser après coup par nos tentatives d'expression, essayant de le figurer en jetant sur lui le filet de nos concepts analogiques. L'être est au contraire, au plus intime de nos attaches - ces « attaches indestructibles » que Merleau-Ponty se plaît à invoquer -, ce qui nous fait apparaître et nous exprime, s'exprimant par là même. Non pas d'abord figuré, mais figuratif, et même archi-figuratif du monde. C'est pourquoi il n'est pas seulement, et pas d'abord, ce vers quoi le visible pointe, un inaccessible à l'horizon (du monde et d'autrui), mais aussi, et d'abord, ce qui "rend visible», cette profondeur ( $\mathrm{du}$ monde, d'autrui et de nous-même) qui nous permet d'accéder au monde, à autrui, et à nous-même.

Où l'on pressent combien cette approche de l'être rompt avec les métaphysiques traditionnelles - à tel point qu'il vaut peut-être mieux, comme Merleau-Ponty le fait lui-même, se contenter de la désigner comme « ontologie »-, et reste irréductiblement liée à une pensée de l'homme. Merleau-Ponty fraye une pensée de l'être qui admet un soubassement et un horizon anthropologiques, fraye une pensée de l'être humain qui admet un soubassement et un horizon ontologiques - il identifie ce chiasme, et l'assume comme tel ${ }^{6}$. Au passage, il

\footnotetext{
${ }^{4}$ Cet être qui «nous porte, qui porte pêle-mêle avec nous d'autres hommes ", et «fait tenir ensemble toutes choses » (M. Merleau-Ponty, La Nature ou le monde du silence, in Maurice Merleau-Ponty, Paris, Hermann, 2008, p. 51/[27](5)-[27]v(6)). La pagination (foliotage) des manuscrits de Merleau-Ponty établie par la B.N.F. est indiquée entre crochets. Elle est suivie, entre parenthèses, de la pagination manuscrite de Merleau-Ponty (lorsque celle-ci existe). La lettre « $\mathrm{V}$ » à la suite du numéro d'un feuillet indique la face verso de ce dernier.

${ }^{5}$ Cf. M. Merleau-Ponty, La Nature ou le monde du silence, op. cit.

${ }^{6}$ Cf. notre ouvrage Vers une ontologie indirecte, Paris, Vrin, 2006.
} 
esquisse à sa manière une co-appartenance de l'homme et de l'être, mais plus encore une co-appartenance des êtres humains dans la portance de l'être.

\section{Aux fondements de notre être-au-monde}

\section{a) Aller à la rencontre du corps et de l'être}

Ce qui en nous s'ouvre à l'être ainsi entendu touche au cœur de l'humanité, car contribue à forger notre identité et nos relations, notre identité relationnelle. Engagés dans notre relation à toute chose, la perception, l'intelligence et le désir participent ensemble à notre ouverture à l'indétermination, à l'épreuve des divers visages de l'être que nous venons d'évoquer, en particulier à celle de sa portance. Ils cheminent dans ces dimensions existentielles dès leur infrastructure corporelle ; aux fondements de notre être-au-monde, ils expriment la vérité du corps humain comme chair ouverte à l'être.

Dans la vie perceptive, le toucher a une situation privilégiée, la plus vitale et la plus existentielle qui soit. En deçà des investissements du désir et de l'incorporation libidinale, il est d'abord sens de l'existence et de la coexistence. Il fait l'épreuve passive-active des limites et de la résistance de ce qui est, de la possibilité de s'appuyer sur lui ; épreuve de la solidité des choses, mais aussi de la cohésion de leurs constituants matériels ; épreuve de la texture et de la contexture des êtres, mais aussi de la qualité et des virtualités de notre lien avec eux, de la puissance de notre corps-à-corps. En continuité avec sa base sensorimotrice, l'intelligence poursuit cette trivalence de la mise à l'épreuve de l'être - l'épreuve de la consistance, celle de la cohésion, celle de l'être-avec. Elle ne commence pas avec la compréhension, mais avec une forme d'étonnement et de consentement interrogatif devant ce qui est. L'intelligence vit et exerce ainsi une double pression - de l'être sur nous et de nous sur lui - en deçà du savoir, en deçà de la possession de déterminations dans la représentation. Elle n'est pas d'abord intelligence de ceci ou de cela, mais une modalité fondamentale de notre ouverture à l'indétermination, capacité d'endurer l'inconnu, d'être en appétit de nouveauté, de s'ouvrir au mystère. À son tour, le désir ne peut être réduit au désir de ceci ou de cela, à l'envie. Il naît en deçà de l'incorporation libidinale, se déploie à travers celle-ci mais aussi au-delà. Non réductible à une émotion, il émerge en deçà de la (possession de telle) détermination, en deçà de l'affect, dans un être à... Au cœur même de l'unité d'animation de notre être, de notre 
manière d'être corps vivant ouvert à l'indétermination, il est d'abord désir d'être, désir de vivre parmi et avec les vivants, d'être corps parmi et avec d'autres corps, d'être avec les choses qui sont. Désir d'être à et avec ce qui est, sans que ce soit d'emblée déterminé comme désir de ceci. D'où sa conjonction originaire avec l'intelligence comme appétit de l'inconnu et de l'invisible, de l'inépuisable - comme appétit de cette profondeur et ces horizons qui sont synonymes de l'être même.

Dégager les fondements de notre être-au-monde nous conduit ainsi à travailler «en deçà »: en deçà de la possession de déterminations, en deçà de la représentation et de l'affect, en deçà de la césure même du cognitif et de l'affectif. Ces « en deçà » nous dirigent conjointement vers le corps et vers l'être (et vers la puissance d'êtreavec du corps humain $)^{7}$. Ils rejoignent les racines de notre intelligence comme épreuve de l'existence et de la coexistence, le cœur de notre animation comme désir d'être et désir de l'être dans l'être-avec. Ils instruisent une conception de la chair comme corps ouvert à l'être, travaillé par l'épreuve de la portance des êtres et la genèse délicate de sa propre portance.

\section{b) Intelligence et épreuve de la portance}

Continuons à esquisser la notion de portance en nous penchant plus spécifiquement sur la participation de l'intelligence aux fondements de notre être-au-monde. Aux prises avec la pression de son environnement, le vivant trouve sans doute sa caractéristique première dans sa capacité d'adaptation. Et c'est avec justesse que Piaget, situant l'intelligence dans son genre, la caractérise elle-même comme adaptation et, en cela, sommet du vivant. Reste à dessiner sa différence spécifique - Piaget le fait en invoquant le couple passif-actif de $\mathrm{l}^{\prime}$ assimilation et de l'accommodation. L'intelligence transforme en partie la structure des choses pour la rendre assimilable, compatible avec la nôtre; elle adapte l'autre à soi pour l'assimiler et qu'il fasse ainsi

\footnotetext{
${ }^{7}$. La philosophie de Merleau-Ponty s'approche à sa manière de ces fondements, dans la phénoménologie d'une vie perceptive aimantée par l'expérience de la coexistence des choses entre elles et avec nous, et dans une opposition farouche à toute pensée réduisant l'être à ses déterminations (l'ontologie de l'objet). Elle s'en approche aussi, sur le tard, par une conception audacieuse de l'inconscient, non freudienne, qui croise comme jamais anthropologie et ontologie, et signe un point d'aboutissement de sa philosophie de la chair, l'inconscient devenant le lieu de rencontre et de communion du corps et de l'être. Voir notre contribution sur «La conception merleau-pontienne de l'inconscient dans les manuscrits tardifs", in Approches phénoménologiques de l'inconscient, M. Gyemant et D. Popa (éd.), Hildesheim/Zürich/New York, Olms, 2015, p. 187-209.
} 
corps avec soi. Symétriquement à cette activité pour ainsi dire digestive, l'intelligence consent à ce que nos structures se laissent en partie configurer et modeler par celles des choses ; comme la vision dont la mise au point obéit à la distance et au grain du perçu, elle accommode. En somme, l'intelligence prend et se laisse prendre, pour faire corps avec l'être connu : elle vit une forme de compréhension, laquelle constitue une forme d'incorporation. Les aventures de cette double structuration réciproque, qui engage un corps-à-corps avec le monde, commence dès l'intelligence sensori-motrice, en deçà de la représentation et du langage.

L'intelligence ne trouve son équilibre et ne progresse que dans la complémentarité de ces deux processus. Une assimilation sans accommodation et une accommodation sans assimilation tombent dans un «activisme » et un " passivisme » également fusionnels et destructeurs. L'approche psychanalytique de l'incorporation enrichit cette description en soulignant qu'une assimilation non destructrice implique aussi une part de rejet, une éjection ou expulsion nécessaire pour éviter la fusion - ce dont ne rendent compte ni l'assimilation ni l'accommodation ${ }^{8}$. L'un des enjeux essentiels de l'adaptation est de survivre en dehors des abîmes symétriques que sont la fusion et la pure séparation, d'édifier des liens non aliénants et de conquérir une identité en n'étant ni tout à fait le même ni tout à fait un étranger. On ne peut en effet tout introjecter ni tout rejeter sans détruire la différence et se détruire soi-même, sombrer dans le vide ou l'aliénation.

D'où l'enracinement existentiel de l'intelligence dans la faculté de juger, dans des proto-jugements d'attribution discriminant ce qui peut faire corps avec nous et ce qui ne le peut pas ou pas encore, différenciant (ce que l'on nomme rétrospectivement, faute de mieux) le «bon» et le «mauvais », l'admissible et le non admissible, dès le corps-à-corps avec la mère. Mais encore faut-il qu'il y ait une mère et qu'elle puisse être vécue comme en partie différenciée, encore faut-il qu'il y ait un autre corps, qu'il fasse corps, et qu'on soit autorisé à le vivre comme en partie autre que le nôtre (ni néant, ni dévorateur). On peut être contraint à la fusion ou condamné à un rejet total - parfois dans une alternance des deux, ambivalence destructrice handicapant l'intelligence dans son rapport au réel et mettant en danger la vie désirante.

\footnotetext{
${ }^{8}$ Par ailleurs, l'accommodation engage elle-même une forme d'incorporation, mais en un sens non psychanalytique : elle n'est à proprement parler ni introjection ni éjection, et s'avère pourtant condition de possibilité d'une incorporation non destructrice. Elle implique une passivité et un abandon, une dimension de consentement essentielle à l'ouverture de la chair à l'être.
} 
Nous avons besoin d'être corps, en corps à corps avec un autre corps; nous avons besoin du corps d'autrui, mais aussi d'un corps commun. Nous avons besoin que cet autre corps ait une dimension de solidité, de consistance et de cohésion, de permanence ou de fidélité pour le dire de la manière la plus simple, qu'il y ait en lui quelque chose qui «tient bon». Ce qui implique qu'il ait une structure, et des limites. Nous avons aussi besoin que cette dimension «solide » nous soit accessible et nous soit prêtée ; et qu'elle le soit sans nous être imposée, en nous laissant le temps et l'espace d'avoir notre propre cohésion et stabilité, notre propre solidité. Nous avons enfin besoin que cet autre corps nous limite; et qu'il le fasse sans nous détruire ni nous rejeter, en nous laissant le temps et l'espace de participer à forger nos propres limites. En somme, nous avons besoin de nous appuyer sur et d'être limité par un autre corps, sans fusion ni séparation totale; mais aussi d'être rattaché et intégré à un corps plus large que le nôtre. Or, l'intelligence est justement convoquée, et au plus haut point, dans l'épreuve de la solidité et de la limite, et dans celle de nos attaches. Dans l'épreuve de la portance.

En deçà de la compréhension et de l'incorporation, l'intelligence fait l'épreuve de la consistance et de la cohésion des choses, de ce en quoi elles sont corps et font corps. En deçà de la représentation, elle $s^{\prime}$ ouvre à la présentation de ce qui est, à la présence des choses. Mais la présence échappe en partie à la prise, elle n'est accessible que dans une certaine inaccessibilité. Résistant à la fusion, sa proximité s'appuie paradoxalement sur un reste de distance, sinon d'absence. Son appréhension n'est pas d'abord préhension, mais épreuve d'un «tenir» aux visages multiples - un minimum de contenance et de résistance, de permanence et de promesse, de persévérance dans l'être.

En deçà de la compréhension et de l'incorporation, et comme condition de leur possibilité, l'intelligence s'enracine ainsi dans l'épreuve de la portance de l'être. Avant que les choses soient compréhensibles et pour qu'elles le soient, encore faut-il qu'elles tiennent dans l'être; encore faut-il aussi que nous tenions nous-même dans l'être; enfin, encore faut-il que nous puissions tenir ensemble dans l'être. Sans cette contenance et cette co-tenance, l'intelligence s'effondre dans le vertige du non-être, du non-sens et du non-lien. Ces trois «tenances» composant la portance ne vont pas de soi (en soi), mais aussi, si l'on peut dire, ne vont pas (de soi) «pour nous»: encore faut-il que nous consentions à ce que ce qui est soit, que nous consentions nous-même à être, et à exister ensemble dans la portance commune de l'être. Il nous faudra revenir sur le jeu serré de ces trois consentements. 
c) Foi et interrogation. Éprouver nos attaches indestructibles

Le dernier Merleau-Ponty s'est penché sur l'interrogation, comme posture fondamentale qui nous anime, soutient toute connaissance et accompagne déjà la perception. Toute question «fait partie de la question centrale qui est nous-mêmes » ${ }^{9}$, celle « de celui qui en est $\gg^{10}$; elle conjoint la saisie de soi à la perception éprouvante de la naissance du monde. Déploiement naturel de l'essence de l'homme dans son Ineinander avec l'être, l'interrogation possède un statut où l'anthropologique et l'ontologique sont indissociables. Merleau-Ponty distingue cette posture interrogative de deux écueils symétriques: le doute radical, et l'attente d'une signification totale qui viendrait combler un vide ${ }^{11}$. Doute absolu ou absolue certitude, ces deux impasses convoquent les fantômes de Descartes et de Sartre, mis en scène dans les déficits du consentement à l'être et du consentement à être, lesquels se rejoignent dans la dénégation de notre lien avec l'être.

L'interrogation est profondément étrangère au négativisme du doute, où "un non être central menace à chaque instant de révoquer son consentement à l'être $»^{12}$. Ce doute est "un positivisme clandestin », analyse finement Merleau-Ponty, il nous faut "le dépasser » vers ce «qu'il nie et qu'il affirme encore ${ }^{13}$. Et si l'on croit pouvoir répondre à notre interrogation fondamentale par "une sphère d'absolue certitude», ce "positivisme des essences » est de son côté habité par «la prétention menteuse de n'être rien ${ }^{14}$, et tombe à nouveau dans une négation de notre inhérence et une coupure $d^{\prime}$ avec l'être. Dans les deux cas, "notre situation de départ est ignorée ${ }^{15}$. L'interrogation n'est pas «recul dans le néant qui n'est rien », car «celui qui questionne n'est pas rien, il est, - c'est tout autre chose -, un être qui s'interroge ; ce qu'il a de négatif est porté par une infrastructure d'être ${ }^{16}$. Nulle question n'est coupée de l'être : « ne fût-ce que par son être de question, elle l'a déjà fréquenté, elle en revient $»^{17}$. Comme l'affirme encore Le visible et l'invisible, "nous sommes des expériences, c'est-à-dire des pensées qui éprouvent la pesée derrière

\footnotetext{
${ }^{9}$ M. Merleau-Ponty, Le visible et l'invisible (désormais noté VI), Paris, Gallimard, 1964, p. 141.

${ }^{10}$ Notes de préparation inédites du Visible et l'invisible (désormais notées NPVI), B.N.F., vol. VII, f. [180]v, [190], [191].

${ }^{11}$ Cf. par exemple VI, p. 160.

12 VI, p. 140.

${ }^{13}$ VI, p. 160.

${ }^{14}$ Idem.

15 VI, p. 161.

16 VI, p. 160.

17 VI, p. 161.
} 
elles de l'espace, du temps, de l'Être même qu'elles pensent ${ }^{18}$. Qui éprouvent la portance de l'être.

L'être s'exprime et nous exprime, s'affirme et nous affirme avant même que nous soyons en mesure de l'interroger: cette précession ontologique donne le cadre matriciel de l'expérience humaine, elle rend possible et provoque notre interrogation. Ni séparés de, ni absorbés par l'être, nous sommes affranchis des deux abîmes, séparation et fusion, qui travailleraient l'analytique sartrienne de l'Être et du Néant. $\mathrm{Ni}$ « coupés de l'être » ni totalement «pris en lui » ${ }^{19}$, nous sommes dans une situation d'empiétement qui configure épistémologiquement nos questions en mystères, et non en problèmes parfaitement solubles ou totalement insolubles ${ }^{20}$. Et c'est bien cette inhérence qui est interrogée. Cet étonnement devant la surprise de l'être, qui nous précède et nous porte, est le principe d'animation de notre intelligence incarnée; cet étonnement intérieur au mystère constitue le fondement existentiel de toute connaissance; il creuse l'écart entre la chair et l'être tout en mettant à l'épreuve leur lien ${ }^{21}$.

En somme, l'interrogation est une manière de «viser $l^{\prime} \hat{E}$ tre ${ }^{22}$, de ressentir et mettre à l'épreuve notre lien avec lui. Son enjeu n'est pas d'abord de nier ni d'être informé, mais d'éprouver une " attache », un « ancrage», et l'éprouver comme "indestructible » ${ }^{23}$. Cette épreuve de nos attaches commence dès la vie perceptive, laquelle n'est pas une simple réceptivité mais fait l'expérience passive-active de la résistance et de la pression des choses ; une expérience pétrie d'agressivité et de désir, de consentement et de foi.

L'analyse merleau-pontienne de la posture fondamentalement interrogative de notre chair met justement en valeur une collusion, subtile et paradoxale, entre interrogation et foi. C'est par un acte de foi que nous nous maintenons hors des abîmes des deux positivismes néantisants dénoncés par Merleau-Ponty, grevés d'un déficit du consentement à l'être et du consentement à être. Nous ne pouvons

\footnotetext{
${ }^{18}$ VI, p. 155.

${ }^{19}$ VI, p. 161.

${ }^{20}$ Cf. par exemple le «Brouillon d'une rédaction » d'octobre 1960 (désormais noté BR), B.N.F., vol. VII, in Notes de cours 1959-1961, Paris, Gallimard, 1996, p. 356/[97](104).

${ }^{21}$ Cette réflexion tardive de Merleau-Ponty sur la pensée interrogative poursuit sa recherche de toujours d'une conception de la connaissance qui sache se démarquer des naïvetés et prétentions de l'idéalisme comme du réalisme (cf. par exemple VI, p. 140). L'aventure de la connaissance s'enracine dans l'épreuve passive-active d'une coexistence, d'un lien de connaturalité, par consentement et participation à la naissance conjointe des choses et de soi. Elle est ainsi «conaissance ", selon la notion que Merleau-Ponty emprunte régulièrement à l'Art poétique de Claudel. ${ }^{22}$ BR, p. 356/[97](104).

${ }^{23}$ Cf. par exemple BR [97](104)-[98](105) (f. non publiés), ou encore VI, p. 160-162.
} 
percevoir sans nous ouvrir à l'indétermination du monde perçu, consentir à accommoder nos structures sensori-motrices à la distance et au grain du sensible, et adhérer à la cristallisation de l'inépuisable. Mais cette foi perceptive possède en son fonds une dimension interrogative préfigurant et fondant toutes nos questions. Car elle n'est pas un consentement purement passif, sinon servile, à la pression et la tension des choses : elle exerce aussi elle-même une pression sur l'être perçu, une mise à l'épreuve d'autant plus radicale que son consentement est fort. La foi interroge et oblige l'être auquel elle se fie et en qui elle se confie. Elle n'est pas adhésion après coup à une identité préalablement délimitée - adhésion au déjà déterminé, au déjà prouvé -, mais consentement à l'indétermination et adhésion à une détermination en cours. Et ceci en partie grâce à cela : sourdement opérante, la foi participe au processus de détermination dans sa manière de consentir à l'indétermination et de l'interroger. La foi passe outre la preuve, éprouve et en cela participe à prouver. Féconde et performative, sa détermination à l'indétermination contribue à une détermination de l'indétermination, comme si sa façon de s'ouvrir à l'inépuisable participait à le cristalliser. Comme si elle contribuait à la réalisation de la promesse à laquelle elle adhère ${ }^{24}$.

Perception, interrogation et foi. C'est bien vers leurs étroites conjonctions et articulations que se dirige la réflexion tardive de Merleau-Ponty à l'occasion de son travail sur Le visible et l'invisible ${ }^{25}$. L'être humain approfondit son «implication inextricable ${ }^{26}$ avec l'être au cœur d'une attente inextinguible et dans une confiance indéfectible ${ }^{27}$ - y compris, sinon d'autant plus, dans l'adversité, " au moment où la vie est menacée ${ }^{28}$. Il l'approfondit dans cette dimension fondamentalement interrogative et éprouvante qui est celle de son existence, du fait même de son attente et de sa foi. Sourdement

\footnotetext{
${ }^{24}$ Cette secrète opérance est au cœur de la logique perceptive que Merleau-Ponty étudie depuis ses premiers travaux, notamment dans la vision en profondeur. La perception s'ouvre à l'indétermination de la profondeur, n'attend pas d'avoir pleinement et parfaitement observé pour cristalliser et reconnaître, mais passe outre la preuve et participe à combler les lacunes du monde perçu, à déterminer l'indétermination. Elle fait l'épreuve d'un écart entre des choses ou dimensions qui ne sont pas préalablement définies, et que cette épreuve même contribue à identifier en les différenciant et les reliant.

${ }^{25} \mathrm{Et}$ ce, dès une note de travail inédite intitulée «Foi perceptive et interrogation ", qui lance deux équations pour le moins audacieuses : d'un côté, entre interrogation et foi, de l'autre, entre perception et interrogation. « Donc interrogation qui est la foi (au lieu de néantiser l'en soi) [...] - Percevoir est interroger: définition d'adaequatio réaliste ou idéaliste rejetée, écart qui est rapport. » (NPVI [162], prob. mars 1959). « La foi est interrogation » (NPVI [163]).

${ }^{26}$ VI, p. 117.

${ }^{27}$ Cf. VI, p. 141 et p. 162.

${ }^{28}$ VI, p. 141.
} 
exercée par la moindre perception, l'interrogation fondamentale qui " porte déjà la philosophie ${ }^{29}$ est ainsi l'épreuve vitale de notre incarnation: $l^{\prime}$ «attache indestructible» par laquelle "nous sommes ancrés dans l'espace et le temps ${ }^{30}$, «le profond mouvement par lequel nous nous sommes installés dans le monde ${ }^{31}$, ce mouvement qui est notre naissance continuée. Elle est aussi au fondement de notre désir de connaître, car l'aventure de la connaissance est la poursuite sans fin de ce besoin existentiel qui est le nôtre "d'éprouver et de comprendre nos attaches $»^{32}$. Enfin, cette épreuve exercée par cet " organe ontologique » qu'est notre foi interrogative, inaugure et accompagne jusqu'au bout notre rapport à l'être ${ }^{33}$.

Nous sommes « une seule question continuée, une entreprise perpétuelle de relèvement de nous-mêmes sur les constellations du monde, et des choses sur nos dimensions $»^{34}$. Une surrection continuée dans laquelle nous nous redressons sans fin sur la portance de l'être et relevons à notre tour les êtres sur les axes porteurs de notre schéma corporel. Dans laquelle l'être que nous sommes et l'être du monde peuvent co-naître, surgir ensemble en un seul être vertical. Tel est ce complexe irréductiblement anthropologique et ontologique où foi, interrogation et épreuve de nos liens ne font qu'un dans l'être percevant et désirant - que Merleau-Ponty esquisse en répondant aux impasses du « complexe ontologique » cartésien et sartrien par l'articulation intime du consentement à être et du consentement à l'être.

\section{Aux fondements de notre être-à-autrui}

\section{a) De l'épreuve de la présence à celle de la relation}

L'étonnement devant la surprise de l'être sollicite en nous les fondements perceptifs et judicatifs de l'intelligence, au cœur d'une dynamique de consentement, de foi et d'interrogation. Nos jugements d'existence, tout en impliquant une perception que ce qui est est, laquelle enveloppe un consentement provisoire à ce que ce qui est soit, ouvrent une interrogation sur et une confiance dans le fait que ce

\footnotetext{
${ }^{29}$ NPVI [190].

${ }^{30}$ BR [98](105) (f. non publié).

${ }^{31}$ VI, p. 141.

${ }^{32}$ BR [98](105) (f. non publié).

${ }^{33}$ VI, p. 162.

${ }^{34}$ VI, p. 140 et voir aussi p. 162.
} 
qui est va continuer à être. Sans s'arrêter à la possession de déterminations dans la représentation ou l'affect, l'intelligence s'ouvre dans une épreuve interrogative de la portance de l'être. Mais il faut pousser l'analyse des fondements de l'intelligence jusqu'à comprendre que cette épreuve est plus fondamentale et plus large que la construction de la fameuse "permanence de l'objet » de Piaget - qui en est une variante déjà cognitivement élaborée, conjointe au premier accès à la représentation. Car elle s'enracine dans l'épreuve de la permanence d'autrui, de la fidélité de sa présence et de sa sollicitude et donc, déjà, dans l'épreuve du lien. Une permanence d'autrui dont Piaget a manqué la spécificité (il l'assimile à la permanence de l'objet), et qui touche à une dimension élémentaire, et fondatrice, de la portance d'autrui.

Le jugement d'existence, envisagé tel que nous le vivons et non dans la rétrospection d'une pure analyse logique, ne se réduit pas à la simple validation ou invalidation d'un «ça existe». Il est aussi et aussitôt jugement de (qualité de) présence et jugement de (possibilité de) relation. L'épreuve de l'être qu'il engage est aussi et aussitôt épreuve de sa coexistence avec nous ; elle convoque inévitablement les enjeux et attentes de la relation, à commencer par ceux de la portance. En cela, nos jugements d'existence sont toujours déjà lourds de protojugements d'attribution, qui s'ouvrent en deçà du langage articulé, et dont la mise en mot rétrospective est délicate ${ }^{35}$. La perception de l'existence s'épaissit aussitôt d'interrogations sur la solidité et la durabilité de celle-ci, sur son "tenir-bon » et ses possibles conséquences. Elle s'oriente d'emblée vers l'épreuve des archi-attributs de la portance même si ce qui est ainsi mis à l'épreuve déborde toujours l'essence vers l'interrogation (de la possibilité et de la qualité) d'une coexistence.

La surprise de la présentation de l'être convoque ainsi une triple interrogation latente, qui tend à mettre en question un "toi », un «moi », et un « entre toi et moi ». Toi. Qu'est-ce, qui es tu ? Question momentanément corrélée à un minimum de consentement à l'être. Moi. Et moi (face à ça, face à toi), quelle place, quelle existence (ai-je, me reste-t-il, reçois-je) ? Question momentanément corrélée à un minimum de consentement à être. Entre toi et moi. Qu'y a-t-il de toi à moi, de moi à toi, entre toi et moi ? Mise à l'épreuve du lien, des

\footnotetext{
${ }^{35}$ La question de l'intrication originelle des jugements d'attribution et d'existence est particulièrement difficile. La faculté de juger n'attend pas la construction du langage et de la représentation pour commencer à se déployer dans l'épreuve de la portance. Or, toute analyse des fondements de l'intelligence les passe au crible de modalités d'exercice de celle-ci qui reposent sur la catégorisation et la pensée logique. Elle ne peut les traduire sans en partie les trahir, souvent en perdant de vue leur enracinement corporel et leurs enjeux relationnels.
} 
attaches (déjà là) comme d'une possible relation (à venir). Question momentanément corrélée à un minimum de consentement à être avec. Chacun de ces trois consentements n'est ici que provisoire, et peut au final échouer, par incapacité à ou refus d'être donné.

Où l'on entrevoit déjà l'implicite de telles formulations, selon deux directions qui convergent dans l'interrogation de la qualité de l'êtreavec. (1) «Est-ce que tu es là pour de bon », " est-ce que tu vas rester là ", " est-ce que tu seras toujours là (pour moi, contre moi, avec moi)». (2) "Est-ce que tu vas me laisser exister, me donner de l'existence », " me laisser ou me donner de l'espace et du temps, une place », « $\mathrm{m}^{\prime}$ accepter, me reconnaître ». Chacune de ces interrogations pouvant envelopper une inquiétude correspondante sur la fragilité de la présence de l'autre, de notre propre être, et de notre relation notamment sur la possibilité de la disparition et de l'abandon, de l'agression et de l'étouffement, du rejet ou de la négation de notre être, du mensonge sur ou du vol de ce que nous sommes ${ }^{36}$. Autant de formulations en germes dès l'étonnement d'une foi interrogative qui met à épreuve, avec l'existence, en elle et à travers elle, la consistance et la permanence, la cohérence et la fidélité de ce qui est.

Sur un plan logique, la question «Et moi ? » (qui suit la question «Toi ? ») semble précéder «Qu'y a-t-il entre toi et moi ? ». Sur un plan anthropologique, cette dernière interrogation est toujours déjà en horizon, tant la relation préside à l'identité, tant la qualité de l'êtreavec porte la formation de ce que nous sommes. À tel point d'ailleurs que la portance reçue allège l'interrogation sur soi, jusqu'à parfois momentanément la lever. Pouvant ainsi déposer et me déposer dans la portance dont l'autre est médiateur, je ne suis plus en question, « je » n'est plus en question, il est abandonné ; je suis sans je, et c'est enfin que je suis.

\section{b) Les gestes de la présentation}

Pour pouvoir s'ouvrir à la portance, encore faut-il endurer la présentation de l'être et s'ouvrir ainsi à une présence qui engage

\footnotetext{
${ }^{36} \mathrm{Si}$ tout jugement d'existence est surdéterminé par les enjeux et attentes de la relation, il est du coup aussi modelé par notre histoire relationnelle et les failles et blessures de celle-ci. Failles et blessures qui se distribuent grossièrement entre trop peu et trop de présence, entre séparation et fusion mortifères, vide de relations et relations destructrices - parfois dans l'alternance voire la concomitance des deux. Mais quelle que soit la tonalité de ces blessures, le tenir-bon reste l'épreuve de base - une relation fusionnelle, étouffante et dévoratrice reste le fait d'un être qui ne tient pas bon et dont la portance, perverse, est déficiente.
} 
toujours déjà une relation. Si nous passons maintenant de la portance reçue à la portance donnée, la notion de présentation demeure opérante, en l'entendant comme un geste posé par celui qui est médiateur de la portance. Un geste qui, en deçà de toute abstraction, peut se déployer selon plusieurs degrés de la relation.

(1) La présentation peut déjà s'entendre comme mise en présence, présence que l'on découvre et qu'on laisse être, voire que l'on accueille et qu'on écoute - ce qui suppose déjà d'éprouver l'autre comme autre, pourvu d'un minimum de consistance. (2) Elle peut aussi signifier une présentation de l'un à l'autre, présentation d'autrui à nousmême et de nous-même à autrui, déjà orientée vers l'interrogation de notre lien. Une interrogation qui passe ainsi, de la découverte de l'autre, à une possible reconnaissance mutuelle, jusqu'à l'éventuel émerveillement devant l'évidence d'un «toi », devant l'évidence de l'être aimé.

(3) La présentation, enfin, peut se déployer dans le sens d'une présentation de l'autre à... À un tiers qui peut être autrui, mais qui peut aussi être indéterminé - présentation de l'autre aux horizons d'un monde et d'un devenir, à l'inépuisable de la vie et de sa vie, à la profondeur de ce qu'il a à être et qu'il est en un sens déjà. Une présentation de l'autre à l'être. Ici, il ne s'agit plus tant d'une présence que l'on découvre ou que l'on reconnaît (sens 1 et 2) que d'une présence que l'on couvre et que l'on ouvre, que l'on protège et nourrit voire que l'on offre, pour qu'elle survive et grandisse. La présentation se fait alors engendrement et offrande, prégnance et naissance de l'autre. Elle marque un accomplissement de la relation dans son ouverture à la transcendance de ce tiers qu'est l'être, un accomplissement du lien dans la portance commune de l'être.

\section{Conclusion. Aux fondements de notre être-à-l'être : la portance commune}

La portance structure la base commune de notre rapport corporel, intelligent et désirant à ce qui est. Son impact se situe en deçà de nos dichotomies usuelles entre le corps et l'esprit, ou encore entre le cognitif et l'affectif - parce que cet impact est existentiel ${ }^{37}$. Dans une tension entre identité et relation, entre existence et coexistence, la

\footnotetext{
${ }^{37}$ En retour, une réflexion sur la portance contribue à interroger ces différenciations, à déstabiliser la pertinence de ces dichotomies qui tendent à se durcir en dualismes.
} 
portance engage les virtualités con-tenantes de l'être, qui peuvent signifier sa capacité à tenir bon, à nous tenir bon (nous contenir), ou encore à nous tenir ensemble. Ce faisant, la portance nous parle du corps, de ce qui fait corps et donne corps à..., non sans lien avec ces trois directions: le corps comme cet autre corps, séparé, résistant et nous résistant, sur lequel nous pouvons nous appuyer (portance); notre propre corps en tant qu'il se tient, nous tient et nous contient (auto-portance); le corps comme ce avec quoi ou avec qui nous sommes, ce avec quoi nous sommes dans l'être-avec, ensemble portés par... (portance commune). Aux fondements de notre être-au-monde et de notre être-à-autrui, la portance sollicite en nous, à nouveau en lien avec les directions de sens que nous venons de rappeler, diverses formes de consentement - à l'être, à être, à être avec. À la racine de notre ouverture à l'être, ces consentements sont engagés dans la posture foncièrement interrogative qui caractérise notre animation et inaugure notre épreuve de ce qui est, dès notre étonnement devant l'existence devant une autre existence, la façon dont elle met en question la nôtre, et interroge notre possible coexistence.

L'articulation de ces trois consentements est particulièrement délicate à analyser, tant chacun d'eux retentit sur les autres, dans une certaine circularité - comme si le consentement à être supposait un consentement à être avec, lequel implique un consentement à l'être qui, en réalité, présuppose déjà un minimum de consentement à être, et à être avec dans l'être. Mais justement, nous touchons ici à la possible priorité d'un consentement qui tend à les conjoindre et à les fonder: un consentement à être avec dans l'être, à l'être-avec de l'être. Un consentement à la portance commune de l'être, sans laquelle rien ne saurait nous apparaître ; à la pesée de l'être derrière nous, sans laquelle il ne saurait pour nous y avoir d'expérience. Nous ne pouvons être sans être avec, nous ne pouvons être sans être avec dans l'être.

Les tensions qui sont celles de l'anthropologie, entre identité et relation, existence et coexistence, autre et avec, trouvent ainsi leur cohérence cachée dans un horizon ontologique: dans une approche de l'être comme portance commune, profondeur et tiers inclus de toute coexistence. Dans cet horizon, la coexistence se redouble: être avec autrui, et être avec autrui dans l'être. Ceci fondant et accomplissant cela. L'être avec autrui est porté par et ouvre sur la transcendance de l'être avec autrui dans l'être. Sans cette portance et ce dépassement, nos relations retombent tôt ou tard dans l'ambivalence de la fusion et de la pure séparation, dans le tourniquet du sado-masochisme, qui brisent la coexistence et replongent nos existences dans une solitude 
destructrice. L'être avec autrui ne peut vivre et survivre que dans la portance commune de l'être. En un sens, seul l'être (seule la portance commune de l'être) est apte à nous faire sortir de la solitude, pas autrui seul ${ }^{38}$.

La portance habite les fondements existentiels de notre être-aumonde (§2) et de notre être-à-autrui (§3) - un être-à-autrui qui n'est pas une région de notre être-au-monde, mais le visage secret de toutes ses dimensions. Mais il faut aller plus loin encore : la portance touche aux fondements de notre être-à-l'être (§1), ceux-ci soutenant ceuxlà. Le monde ne suffit pas (à nous porter), autrui même non plus, toute portance s'enracine dans celle de l'être. Dans une portance commune qui soutient secrètement toutes nos relations, et donne à l'éthique son nécessaire enracinement ontologique.

\footnotetext{
${ }^{38}$ Sans pouvoir ici aborder les sources et enjeux cliniques de cette réflexion sur la portance, signalons néanmoins que nous la travaillons dans divers dispositifs complémentaires aux modes d'accompagnement psychothérapique classiques. Ces dispositifs, notamment celui de l'atelier (de l'artisan ou de l'artiste), axés sur l'ouverture à la portance commune de l'être, ne sollicitent pas d'abord la parole mais les (autres) gestes du corps, et favorisent un rapport à autrui qui n'est pas le face à face de la plupart des psychothérapies ni le « face à dos » du divan, mais un côte à côte passant par une confrontation commune à la matière ou à la nature, dans une adversité partagée, dans une forme d'intercorporéité articulée sur un tiers inclus qui n'est pas une personne humaine - mais la matière à façonner, le bâtiment à construire, le terrain à cultiver, la montagne à gravir, le désert à traverser, etc.
} 